\title{
Gesundheitsziele haben die Erhaltung oder Verbesserung von Gesundheit zum Ziel
}

\section{Liebe Leserinnen und Leser,}

zwar ist obige Erklärung von Gesundheitszielen im Grunde trivial, sie hat aber mindestens 2 Vorzüge: Zum einen kann man bei dieser Definition $\mathrm{Ge}$ sundheitszielen nur positiv gegenüber stehen. Zum anderen wird unweigerlich Neugierde geweckt: Wer kümmert sich wie um die Erhaltung oder Verbesserung von wessen Gesundheit?

Da der Jahreswechsel noch nicht so weit zurück liegt, wird jeder von Ihnen seine individuellen $\mathrm{Ge}$ sundheitsziele für das Jahr 2003 noch im $\mathrm{Ge}$ dächtnis haben: Dazu gehören meist Vorsätze wie mehr Sport zu treiben, gesünder zu essen, weniger Alkohol zu trinken, sich das Rauchen abzugewöhnen. Es gehören auch Wünsche dazu, deren Erfüllung nicht (allein) in unserer Macht liegt: Wer krank ist, möchte optimal behandelt und wieder gesund werden. Und wohl jeder wünscht sich Gesundheit auch für all seine Verwandten und Freunde.

Wenn Gesundheit ein so hohes Gut ist, wieso sind dann Gesundheitsziele keine Selbstläufer? Es ist nur ein Teil der Antwort, dass die guten Vorsätze über das Jahr meist in Vergessenheit geraten oder nur mit Unterstützung von außen umgesetzt werden. In den meisten Fällen sind individuelle Gesundheitsziele auch so komplex, dass sie sich nicht allein mit dem guten Willen umsetzen lassen: Es bedarf des Wissens um
Zusammenhänge, der Kompetenz im Umgang mit Gesundheit und Krankheit, es braucht die Unterstützung im familiären und sozialen Umfeld. Es bedarf aber auch der Versorgungsangebote im Krankheitsfall, die evidenzbasiert und dennoch individuell auf den $\mathrm{Pa}$ tienten zugeschnitten (und finanzierbar) sind.

Es darf zudem nicht übersehen werden, dass viele Menschen zwar Gesundheit schätzen, ihren eigenen Anteil an deren Erhaltung aber nicht sehen. Wie ließe es sich sonst erklären, dass die Bundeszentrale für gesundheitliche Aufklärung kürzlich feststellen musste, dass der Anteil rauchender Jugendlicher weiter zugenommen und sich das Einstiegsalter für das Rauchen weiter verringert hat? Was ist in diesem Fall mit den Gesundheitszielen? Werden sie von anderen umgesetzt und etwa gegen die Einsicht der Betroffenen? Darf jemand anderes als Sie selbst ein Gesundheitsziel umsetzen, das Ihre eigene Gesunderhaltung zum Ziel hat? Wenn außer Ihnen auch noch Krankenkassen, Gesundheitsministerien, Rentenversicherer und Gesund-

\footnotetext{
C) Springer-Verlag 2003

Dr. Bärbel-Maria Kurth,

Robert Koch-Institut, Abteilung für Epidemiologie und Gesundheitsberichterstattung, Seestraße 10,13353 Berlin,

E-Mail:kurthb@rki.de
} 


\section{Editorial}

heitswissenschaftler Interesse an Gesundheitszielen und damit auch an Ihrer Gesundheit haben, sollten Sie dann eher misstrauisch oder glücklich sein? Was ist, wenn Ihr individuelles Gesundheitsziel nicht zu den prioritären nationalen Gesundheitszielen zählt? Sollen mit Gesundheitszielen letztendlich Ausgaben vermieden werden oder kann dafür zusätzlich Geld aufgebracht werden? Und wieso begrüßt das Robert Koch-Institut als für die Gesundheitsberichterstattung des Bundes zuständige Institution die Entwicklung von $\mathrm{Ge}$ sundheitszielen?

Ich hoffe, Sie sind nun ausreichend motiviert, dieses Schwerpunktheft möglichst in der vorgegebenen Reihenfolge der Artikel zu studieren. Ich verspreche Ihnen nicht nur ein „Aha-Erlebnis“. Eine gute theoretische Basis und viel strukturelles Verständnis für die Notwendigkeit und das Procedere der Entwicklung von Gesundheitszielen schaffen der internationale Vergleich und der sehr systematische Einstieg in die Entwicklung von nationalen Gesundheitszielen zu Beginn des Heftes. Das Setzen objektivierbarer Kriterien für die Auswahl prioritärer Gesundheitsziele, das Aufeinanderabstimmen verschiedener Maßnahmen zur Erreichung desselben Zieles, die immer wiederkehrende Beschreibung der Konsensusfindung bei Beteiligten aus sehr unterschiedlichen Interessengruppen und die Gedanken über die Evaluation machen die Arbeiten zu den 5 Themenbereichen der nationalen Gesund- heitsziele für den etwas eingeweihten Leser zur spannenden Lektüre.

Es könnte allerdings auch der ketzerische Gedanke „Die Bewegung ist alles, das Ziel ist nichts“oder auch „Der Weg ist das Ziel" aufkommen. Aber in Deutschland sind Gesundheitsziele schon sehr viel weiter als die grundsätzliche Diskussionsebene entwickelt. Das zeigt der Beitrag aus Nordrhein-Westfalen, der sehr plastisch darstellt, wie in einem Bundesland bereits sehr konkrete Ergebnisse bei der Umsetzung von $\mathrm{Ge}$ sundheitszielen erreicht wurden. Positive Beispiele zur Umsetzung kommunaler Gesundheitsziele gibt es noch weitaus mehr, wenn sie auch aus Platzgründen nicht in diesem Heft dargestellt werden können. Das bereits Erreichte stimmt optimistisch, was die Umsetzung der ersten 5 nationalen „Gesundheitszielbündel" betrifft.

Wünschen wir uns also für das Jahr 2003, dass wir sowohl den individuellen als auch den regionalen, den kommunalen wie den nationalen Gesundheitszielen ein Stück näher kommen. Schließlich gilt für das Wesen von Zielen: „Ein Werk ist erst dann vollendet, wenn das Ziel erfüllt ist" (Paulo Coelho, „Der Alchimist“).

Ihre

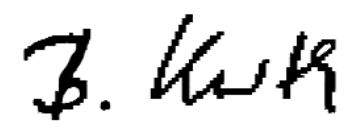

Bärbel-Maria Kurth 